\title{
Infectious disease, the innate immune response, and fibrosis
}

\author{
Alessia Meneghin and Cory M. Hogaboam \\ Immunology Program, Department of Pathology, University of Michigan Medical School, Ann Arbor, Michigan, USA.
}

\begin{abstract}
The unrelenting and destructive progression of most fibrotic responses in the pulmonary, cardiovascular, integumentary, and alimentary systems remains a major medical challenge for which therapies are desperately needed. The pathophysiology of fibrosis remains an enigma, but considerable research and debate surrounds the question of whether chronic inflammation is the key driver of unrestrained wound healing (i.e., the fibrotic response) in these and other organ systems. This Review describes how infectious pathogens, chronic inflammation, and unrestrained fibroproliferation are likely to be part of a dynamic, unrelenting process propelling human fibrotic diseases.
\end{abstract}

\section{Tissue repair: to heal or pathologically scar}

Tissue repair normally occurs very quickly after mechanical trauma, thermal trauma, or infection causes tissue injury. The resolution of tissue injury involves sequential and well orchestrated phases of immune cell recruitment and formation of granulation tissue; secretion of growth factors, cytokines, and chemokines; formation of new ECM; angiogenesis; and, finally, the return of the tissue to its pre-injury state (1-4). Fibroblast activation and proliferation are key components in the tissue repair process, and activation of these cells is characterized by the de novo production and deposition of ECM proteins such as collagen type I, collagen type III, fibronectin, elastin, proteoglycans, and laminin $(5,6)$. A delicate balance between ECM deposition by fibroblasts and ECM degradation by recruited leukocytes is necessary for a healthy outcome during the tissue repair process (7). Several MMPs and tissue inhibitors of metalloproteinases (TIMPs) are expressed during wound healing and control ECM remodeling by degrading existing ECM in and around the wound edge and by creating a path for cell migration as new ECM is deposited (8). The balance between ECM formation and degradation is key during the normal wound healing process. Indeed, ECM degradation leads to the generation of protein fragments that provide important biological activities needed to facilitate normal tissue repair at an injury site (8). A dynamic balance between proliferation and apoptosis normally regulates the number of fibroblasts at an injury site and thereby regulates ECM turnover (9). All of these processes normally combine to return tissues and organs to their pre-injury state, able to function without impairment.

Should any feature of this elaborate resolution process be disrupted or remain overly exuberant, abnormal tissue repair or pathological scarring occurs $(5,9,10)$. In most human tissues, a pathological consequence of an inappropriate or poorly regulated tissue repair process is described as fibrosis $(1,5,11-15)$. Fibroproliferation is a complicated montage of various mechanisms

Nonstandard abbreviations used: ARG1, arginase 1; CCL, CC chemokine ligand CXCL, CXC chemokine ligand; HHV8, human herpesvirus 8; IPF, idiopathic pulmonary fibrosis; MHV-68, murine $\gamma$-herpesvirus-68; NLR, nucleotide-binding oligomerization domain-like receptor; NSIP, nonspecific pneumonia; PAMP, pathogen-associated molecular pattern; PRR, pattern recognition receptor; RIG-I, retinoic acid-inducible gene I; TIMP, tissue inhibitor of metalloproteinases; vGPCR, viral $\mathrm{G}$ protein-coupled receptor.

Conflict of interest: The authors have declared that no conflict of interest exists. Citation for this article: J. Clin. Invest. 117:530-538 (2007). doi:10.1172/JCI30595. involving the sustained interaction between activated immune and structural cells, a myriad of soluble and cell-associated biological factors, pronounced impairment of tissue or organ function, and perhaps even death of the affected individual. The main subject of this Review is that the persistence of exogenous and endogenous stimuli provided or induced by infectious pathogens such as bacteria, viruses, fungi, and multicellular parasites is a major promoter of fibrosis in many organs, including those associated with the pulmonary, cardiovascular, integumentary, and alimentary systems (Figure 1). A partial listing of major microorganisms that have been associated directly and/or indirectly with organ fibrosis is summarized in Table 1 . The persistence of these infectious stimuli and the sustained injury that results might drive fibrosis because their presence induces marked alterations in a number of immune and structural cells. This Review highlights experimental and clinical findings surrounding the unique activation status of fibroblasts and macrophages in fibrotic lesions characterized by the presence of some of these pathogens and/or their byproducts (Figure 1).

\section{Important cellular culprits of fibrosis}

Fibroblasts. The fibroblast clearly has a necessary and fundamental role in tissue homeostasis and normal wound repair through its production of ECM proteins (16). However, this cell is clearly also the culprit in chronic fibrosing diseases: their dynamic and seemingly unregulated activity during chronic inflammatory responses is well documented (17), and activated fibroblasts, often demarcated by their increased expression of $\alpha$-SMA (hence the term "myofibroblast"), are present in fibrotic tissue (18). Fibroblasts exhibit heterogeneity (in terms of their cellular phenotype and origin) in $(19,20)$ and between different organ systems $(21)$. The developmental origin of fibroblasts is not disputed - they are of mesenchymal origin during embryonic organogenesis. However, there is growing evidence that these cells might be derived from other cellular sources in the adult (22). Several groups have compelling evidence that new fibroblasts can arise in the lung, liver, and kidney from epithelial structures as a result of a process known as epithelialmesenchymal transition (reviewed in ref. 23). Other studies indicate that BM-derived fibrocytes represent another potential cellular origin for collagen-producing cells $(24,25)$. These cells seem to gain entry to damaged tissues such as the skin and lung through chemokine-dependent mechanisms $(26,27)$. Once in these tissues, BMderived fibrocytes seem to mature into myofibroblasts and secrete 


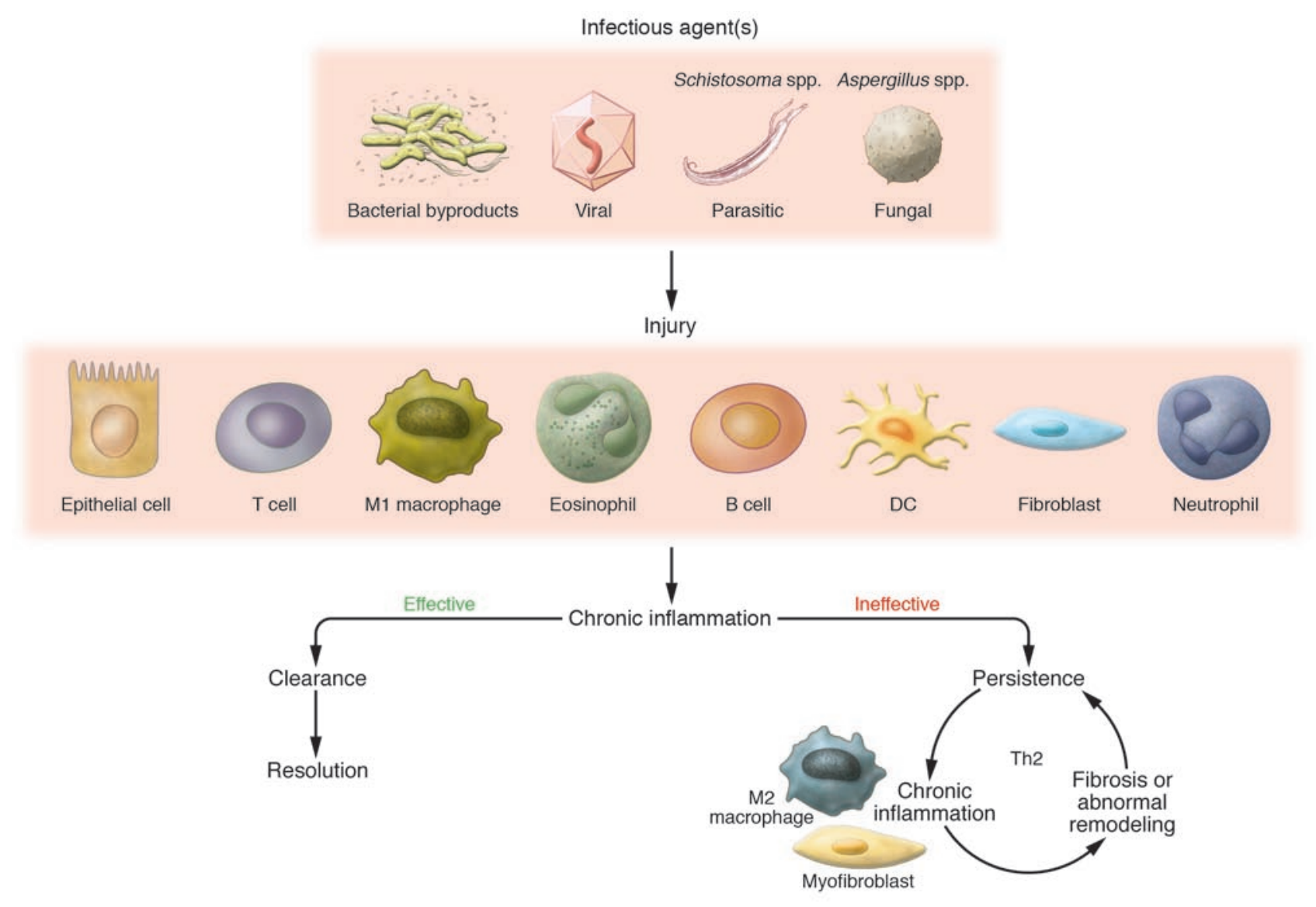

Figure 1

Persistent infectious stimuli initiate and sustain the fibrotic process. Pathogens such as bacteria, viruses, fungi, and multicellular parasites represent major tissue injurious signals. The host response, mediated by the innate immune system, is intricately directed toward recognizing PAMPs through a myriad of PRRs. The concerted inflammatory and immune efforts of several immune and nonimmune cell types, including (but not limited to) classically activated macrophages (M1 macrophages), T cells, eosinophils, B cells, DCs, neutrophils, epithelial cells, and fibroblasts, contribute to the chronic inflammatory response. A dominant Th1 cytokine response normally characterizes an effective immune response against most pathogens, except most multicellular and extracellular parasites. Should pathogens and their byproducts be effectively cleared, the affected tissue often heals appropriately and the inflammatory process resolves. However, pathogens use various survival strategies to avoid elimination, and this leads to their persistence or the persistence of their byproducts in the host. The cytokine pattern associated with the ineffective response is often skewed toward the Th2 cytokine pattern. The resulting fibrotic response might be a consequence of the actions of unique cells such as the ECM component-synthesizing myofibroblast and the alternatively activated macrophage (M2 macrophage). The fibrotic response, in turn, might facilitate the persistence of pathogens and their byproducts, thereby allowing this vicious cycle to continue.

ECM proteins to promote wound healing (28). Therefore, in the adult, fibroblasts seem to be derived from at least three sources: locally by proliferation or transdifferentiation and distally from the BM. Current research challenges involve effectively demarcating the relative quantity of each fibroblast type in a given fibrotic lesion and determining whether one, two, or all three of these sources of fibroblasts actually contribute to the pathology of fibrosis during chronic infectious diseases. This challenge is particularly daunting in light of the absence of distinct lineage-specific markers associated with each fibroblast type.

Macrophages. Activated macrophages have a prominent role in the innate immune response to infection and/or tissue injury through their ability to phagocytose particles such as bacteria and to secrete proinflammatory cytokines. It was initially thought that all macrophages were active during inflammation, due to the presence of proinflammatory stimuli and Th1 cytokines, and quiescent during tissue resolution, due to the presence of glucocorticoids and
Th2 cytokines such as IL-4 and IL-13 $(29,30)$. However, several studies now show that macrophages are alternatively activated under conditions predominated by Th2 cytokines, so that, similar to Th1and Th2-polarized T cells, macrophages activated in the presence of Th1 and Th2 cytokines have distinct phenotypes. In particular, classically activated macrophages produce large amounts of oxygen free radicals that participate in the acute inflammatory process. As alternatively activated macrophages synthesize and/or induce the production of many profibrotic factors, this macrophage subtype is strictly related to tissue fibrosis in the course of inflammation (29). Mantovani et al. (30) have proposed a nomenclature system for polarized macrophages, referring to classically- and alternatively-activated macrophages as M1 and M2, respectively. Classically activated (M1) macrophages result from exposure to the Th1 cytokine IFN- $\gamma$, LPS, and TNF- $\alpha$, and as producers of large amounts of IL-12 and NO, they effectively kill various microbial pathogens. M1 macrophages are the prototypical macrophages characterized by features such as phago- 
Table 1

Main pathogens associated directly and/or indirectly with organ fibrosis

Microorganism

M2 macrophages
present and/or
involved?

Target organ

Reference

involved?

\begin{tabular}{|c|c|c|c|}
\hline \multicolumn{4}{|l|}{ Bacteria } \\
\hline Mycobacterium tuberculosis & yes & L, Li, Sp, B, K & 43 \\
\hline Mycobacterium leprae & yes & L, Li, Sp, S & 115 \\
\hline Pseudomonas aeruginosa & no & $\mathrm{L}, \mathrm{Gu}$ & 116 \\
\hline \multicolumn{4}{|l|}{ Viruses } \\
\hline HCV & no & $\mathrm{Li}$ & $63,65,66$ \\
\hline HBV & no & $\mathrm{Li}$ & 117 \\
\hline Respiratory syncytial virus & no & L & 118 \\
\hline Herpesviruses (EBV, CMV, HHV8, MHV-68) & yes & L, Li, K, B & $69-74,119$ \\
\hline \multicolumn{4}{|l|}{ Parasites } \\
\hline Schistosoma mansoni & yes & L, Li, Sp, H & $103-105,112,120-122$ \\
\hline Toxoplasma gondii & yes & L, Li, Sp, B, K & 113 \\
\hline Pneumocystis carinii & yes & $\mathrm{L}$ & 123 \\
\hline Cryptosporidium parvum & yes & $\mathrm{L}, \mathrm{G}$ & 114 \\
\hline Leishmania spp. & yes & Li, Sp, G, S & 113 \\
\hline Trypanosoma spp. & yes & $\mathrm{H}$ & 113 \\
\hline Trichinella spiralis & unknown & $\mathrm{Gu}, \mathrm{H}, \mathrm{L}$ & 113 \\
\hline Brugia malayi & yes & $\mathrm{Gu}$ & 113 \\
\hline Taenia spp. & yes & $\mathrm{Gu}, \mathrm{H}$ & 113 \\
\hline \multicolumn{4}{|l|}{ Fungi } \\
\hline Aspergillus fumigatus & yes & $\mathrm{L}$ & $81,83-85,90-98$ \\
\hline Candida albicans & yes & $\bar{L}$ & 124 \\
\hline Cryptococcus neoformans & yes & $L, B$ & 125 \\
\hline
\end{tabular}

B, brain; G, gut; Gu, genito-urinary tract; H, heart; L, lungs; Li, liver; K, kidneys; S, skin; Sp, spleen.

bolic, and immunological factors, represent important mechanisms of defense too, by influencing the efficiency of the immune response. A combination of physical (such as an epithelial barrier), chemical (such as mucoid and protein secretions), and immunological defenses (such as tissue-resident macrophages and DCs) is particularly active at sites where injury and/or infections pose the greatest threat - organs that are exposed to the external environment, such as the skin, gut, lungs, and genito-urinary tract. In general, the initial host response to a pathogen is rapidly generated by the innate immune system, and this response often leads to the successful elimination of the microorganism. This rapid response system largely depends on a sophisticated array of pattern recognition receptors (PRRs), which recognize conserved pathogen-associated molecular patterns (PAMPs) (34). Three major families of PRRs have been discovered: intracellular and extracellular TLRs $(35,36)$, intracellular nucleotide-binding oligomerization domain-like

cytosis, antigen processing and presentation, and $\mathrm{T}$ cell activation. More importantly, M1 macrophages express iNOS (also known as NOS2), which metabolizes L-arginine to generate the large amounts of $\mathrm{NO}$ that are involved in intracellular pathogen killing. Alternatively activated (M2) macrophages secrete the regulatory cytokines IL-10 and TGF- $\beta$ as well as the soluble IL- 1 receptor antagonist (IL-1Ra) and express the type II IL-1 decoy receptor on their surface (30). All these characteristics enable M2 macrophages to efficiently inhibit the M1-driven inflammatory process. This is due, in part, to the fact that these cells express high levels of arginase 1 (ARG1), which directly competes with iNOS for L-arginine. M2 activation results in increased expression of factors that cause tissue fibrosis (that is, TGF- $\beta$, fibronectin, proline, and TIMPs) (31) and cytokines such as IL-10 and CC chemokine ligand 17 (CCL17). CCL17 binds CC chemokine receptor 4 (CCR4), and the interaction between these two has been shown to drive fibrogenesis in several mouse models of pulmonary disease $(32,33)$. In healthy individuals, $M 2$ macrophages are predominantly found in the placenta and lung, where they promote tolerance to self antigens and curb inflammation, but these cells have an increasingly understood role in pathologic processes such as tumorigenesis, asthma, allergy, and fibrosis (30).

\section{From pathogens to the chronic inflammatory response and pathological tissue fibrosis}

Although the nature of the infecting pathogen influences the type of immune response that the host generates, many endogenous and exogenous factors, including genetic, physical, chemical, meta- receptors (NLRs) (37), and intracellular retinoic acid-inducible gene I (RIG-I) receptors (38). Many cells of the immune system, including macrophages, T cells, eosinophils, B cells, DCs, NK cells, mast cells, and neutrophils, express PRRs, but these receptors have also been detected in structural cells such as fibroblasts, epithelial cells, and adipocytes (39). PAMPs include pathogen byproducts such as lipoproteins and lipopeptides, peptidoglycans, lipoteichoic acid, LPS, double-stranded RNA, flagellin proteins, and hypomethylated DNA $(15,37,40)$. More recently, it has been shown that TLRs can also recognize endogenous proteins that have been modified by oxidation or nitration (41) as well as heat shock proteins that are released during acute inflammation and from damaged or dying cells (42). It is plausible that the cooperation of several PRRs in the recognition of PAMPs initiates the first line of defense against infection and/or injury, including the production of proinflammatory cytokines, the synthesis of chemotactic cytokines that recruit cells of the immune system, enhanced phagocytosis, and the release of antimicrobial peptides (36). The intricate interactions between the host and pathogen dictate the natural history of many infectious diseases, and the persistence of many infectious pathogens is a consequence of the ability of these microorganisms to modulate the immune response through various ingenious effector mechanisms (36). The failure to clear the pathogen and/or its byproducts consequently provides a persistent source of tissue injury and chronic inflammation. This results in fibrosis, which is therefore an undesirable outcome of an overactive innate immune system. 
Pathogen byproducts modulate the fibrotic process. Little is presently known about the expression and role of PRRs in the progression of bacterially induced chronic fibrosing diseases such as pulmonary tuberculosis, which is characterized by the presence of M2 macrophages and activated fibroblasts in lesions or granulomas that contain Mycobacterium tuberculosis (43). Similar to M1 macrophages, M2 macrophages express TLRs, but activation through these PRRs seems to promote M2 macrophages to produce cytokines and chemokines such as IL-10 and CCL17, as opposed to the proinflammatory stimuli produced by M1 macrophages (44). IL-10 and CCL17 work to limit the inflammatory response of M1 macrophages (45). One intriguing explanation for the inappropriate tissue repair that causes fibrosis during persistent bacterial infections (such as pulmonary tuberculosis) might be that tissue-resident structural cells acquire and maintain the ability to respond to antigen-independent danger signals normally reserved for cells of the immune system (46). Generally, expression of PRRs by immune cells such as M1 macrophages serves a protective role following infection. Structural cells such as type II alveolar epithelial cells (47), airway smooth muscle cells (48), airway epithelial cells (49), and fibroblasts express TLRs, including TLR2, TLR3, TLR4, and TLR9, and are activated by specific TLR ligands. Less is known about the expression and activation of other PRRs in these cell types. The activation of TLRs in structural cells might also contribute to the immune and antiinflammatory processes during infection but, as described below, TLR activation in these cells might lead to pathological tissue scarring.

Emerging data suggest that PAMPs might provide a major activating signal to fibroblasts through TLRs and possibly through NLRs and RIG-I. For example, myofibroblasts isolated from the gut of patients with Crohn disease showed robust expression of TLR2, TLR3, TLR4, TLR6, and TLR7 and expressed MyD88, which is the adaptor molecule that links many TLRs to their downstream signaling pathways (50). A major consequence of TLR activation of gut myofibroblasts was the secretion of CXC chemokine ligand 8 (CXCL8). CXCL8 was initially characterized as a neutrophil chemotactic factor, but its biological properties extend to angiogenesis and fibrosis (51). Chemokine secretion has also been studied in the context of rheumatoid arthritis synovial fibroblasts (52). Following TLR2 activation by peptidoglycan, these primary fibroblasts released large amounts of CC and CXC chemokines including CCL5, CCL8, and CXCL6 (52). In an experimental mouse model of myocardial infarction, TLR2 was found to exert a major role on the development of myocardial fibrosis but not on the size of the infarct or the degree of inflammatory cell infiltration into the infarct (53). Fibrogenesis and the consequent ventricular tissue remodeling is a major complication following myocardial infarction, and this process contributes to long-term cardiac dysfunction and mortality in this patient population (54). Intracellular TLR3 activation by double-stranded RNA caused synovial fibroblasts to produce CXCL10 (55), and CCL5 generation by a respiratory syncytial virus-infected fibroblast cell line was induced directly by TLR3 signaling pathways (56). In an experimental in vivo system, TLR9 activation by CpG DNA was shown to drive interstitial fibrosis during lupus nephritis (57). More recently, TLR activation of embryonic and transformed fibroblast cell lines led to sensitization for apoptosis (58) and stimulated cell cycle entry (59), respectively. Whether these consequences of TLR activation apply to primary fibroblasts during the resolution of tissue injury and/or the development of pathological tissue fibrotic scarring has yet to be determined, as has their role in the development of fibrosis.

Preliminary unpublished data in our laboratory have revealed intriguing differences in TLR expression and activation between normal fibroblast lines and those derived from patients with severe pulmonary fibrotic forms of idiopathic interstitial pneumonia (idiopathic pulmonary fibrosis [IPF] and nonspecific pneumonia [NSIP]). For example, we have observed that TLR9 activation through hypomethylated DNA in fibroblast lines derived from lung biopsies of patients affected with IPF and NSIP promotes the de novo generation of profibrotic signals such as CCL2 (our unpublished findings). CCL2 is a potent profibrotic chemokine, levels of which have been shown to be elevated in the context of various fibrotic diseases, including those affecting the renal (60), cardiovascular (61), and pulmonary (62) systems. Therefore, compelling evidence shows that fibroblasts from various chronically diseased tissues can express TLRs and respond to PAMPs in a manner that can activate fibroblasts during inappropriate fibroproliferative responses. Together, these findings provide clear impetus for pursuing studies directed at further elucidating the effects of TLR ligands on cytokine-activated fibroblasts from various tissue sources.

Persistent viral infections promote and enhance fibrosis. Viruses are highly disruptive to tissues by nature of their cytopathic effects, particularly during the lytic phase of viral replication. Associated with the digestive system, HCV is one example of a virus that chronically infects hepatocytes, causing chronic inflammation that can lead to organ fibrosis. Liver pathology during the course of $\mathrm{HCV}$ infection is characterized by portal tract and lobular mononuclear cell inflammation (63), and the recurring tissue repair in the liver parenchyma involves fibrogenesis leading to the loss of organ architecture and function (64). The collagen and ECM proteins that are required for fibrosis to occur are largely produced by activated hepatic stellate cells that have taken on a myofibroblast phenotype (14). The presence of activated fibroblasts in the HCVinfected liver is a direct consequence of increased amounts of the highly profibrotic mediator TGF- $\beta$, derived from cells rendered necrotic during the lytic phase of $\mathrm{HCV}$ infection (65). $\mathrm{HCV}$ is also able to directly promote fibrogenesis, as some of the HCV core proteins promote hepatocytes to secrete oxygen free radicals (66) and block normal liver regeneration (67).

Both the lytic and latent phases of the life cycle of a virus can provide major stimuli for tissue fibrosis. During viral latency, a small percentage of infected cells, including fibroblasts, can express distinct viral antigens on their cell surface. In several organs, including the lung, the persistence of these and other foreign antigens promotes the development of a Th 2 cytokine environment $(68,69)$. Within this polarized Th2 tissue environment, the organ seems to be at particular risk for a secondary viral infection, and this second hit might then trigger amplification of innate immune and tissue remodeling events (Figure 2).

Debate surrounds the contribution of viruses to clinical fibrotic diseases. EBV and certain herpesviruses, such as human herpesvirus 7 (HHV7) and HHV8 (also known as Kaposi sarcoma-associated herpesvirus), have been detected in patients with IPF, the most common (and, alas, severe) form of primary pulmonary fibrosis (70). Interestingly, alveolar content samples from patients with IPF contain M2 macrophages $(71,72)$, and it has been suggested that the upregulation of the alternative activation pathway in these cells might contribute to the fibrotic process because these cells 

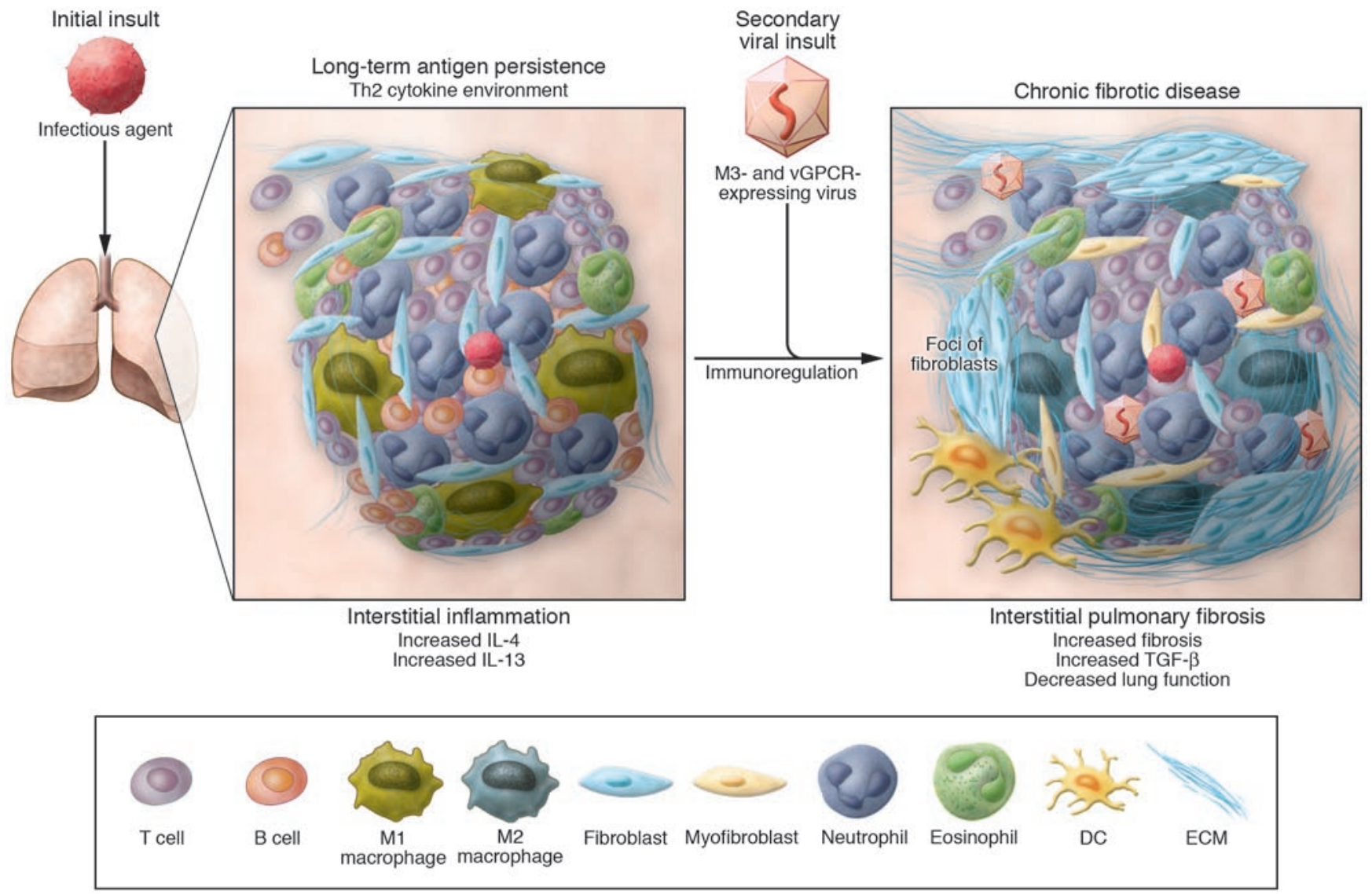

Figure 2

A defective host response facilitates chronic pulmonary fibrosis. Similar to other organ systems directly exposed to the external environment, the lung confronts infectious agents on a continual basis. Should transcript and protein components of these infectious pathogens persist, the immune response appears to skew to a Th2 cytokine profile (i.e., increased IL-4 and IL-13). Secondary or repetitive exposure to infectious agents in this immunoregulated environment might then exacerbate the fibrotic response due the lack of an effective Th1 immune response. This scenario can be observed in the context of a secondary viral insult in the form of a herpesvirus. Experimentally, MHV-68 markedly enhanced fibrosis in a Th1-deficient environment (73). Current data suggests that MHV-68 exacerbates the tissue fibrotic response in mice by contributing gene products that can neutralize necessary innate immune cytokines (through cytokine-binding proteins such as M3) or induce rapid cell proliferation (through the viral chemokine receptor vGPCR) (our unpublished observations). Therefore, the fibrotic response might be exacerbated by secondary and repeated pathogen insults. Viral byproducts might be particularly important in perpetuating fibrosis.

inhibit the pulmonary antiviral response (72). When mice lacking the receptor for IFN- $\gamma$ (Ifngr ${ }^{/-}$mice) were challenged with the murine homolog of HHV8, murine $\gamma$-herpesvirus-68 (MHV-68), they exhibited multi-organ fibrosis (73). The development of multi-organ fibrosis in these mice might reflect the persistence and dissemination of this virus within this knockout mouse, which has an immune response heavily skewed toward a Th2-type cytokine response. More recently, it has been shown that chronic infection of Ifngr ${ }^{-1}$ mice with MHV-68 promotes the recruitment of macrophages into the lung, and these cells undergo alternative activation (72). Although the examination of fibrotic events in a mouse completely lacking the potent antifibrotic effects of IFN- $\gamma$ signaling can be interpreted as contrived, other investigators have shown that MHV-68 promotes bleomycin-induced pulmonary fibrosis in BALB/c mice, a mouse strain that is normally resistant to the profibrotic effects of this anti-neoplastic antibiotic (74). One explanation for the appearance of fibrosis in mice concomitantly challenged with bleomycin and MHV-68 is that infection with MHV-68 skews the pulmonary immune response toward a Th2- type cytokine pattern, which results in dysregulation of the repair process (possibly through alternative activation of macrophages) and the development of pathologic lung scarring.

Current data from our laboratory suggest that MHV-68 exacerbates the tissue fibrotic response in mice by contributing gene products that can neutralize specific proinflammatory cytokines (for example, the cytokine-binding protein M3; Figure 2) or induce rapid cell proliferation (for example, the constitutively active viral G protein-coupled receptor [vGPCR]; Figure 2) (our unpublished observations). Similar mechanisms might occur in clinical fibrosing diseases. For example, open reading frame 74 of HHV8 encodes vGPCR, which is a constitutively active chemokine receptor involved in the tumorigenesis and cytokine production associated with Kaposi sarcoma (75). In cells transfected with vGPCR, the constitutive activity of this receptor induces the expression of proinflammatory cytokines (such as IL-1 $\beta$, IL-6, and TNF- $\alpha$ ), chemokines (such as CXCL2 and CCL2), and growth factors (such as FGF- $\beta$ ) (76). Our quantitative PCR analysis has shown that mRNA encoding the HHV8 vGPCR is present in the biopsy and fibroblast 
cultures derived from patients with either UIP or NSIP (our unpublished observations). Furthermore, we have observed that it is expressed at markedly higher levels in these samples than in lung biopsies and human fibroblast cell lines derived from healthy individuals (our unpublished findings). Therefore, further investigation of the profibrotic implications of the expression of vGPCR and other gene products by viruses that establish a persistent infection in immune and structural cells is certainly warranted.

Persistence of fungal antigens and peribronchial fibrosis. Asthma is characterized by a Th 2 cytokine-driven airway inflammatory response that encompasses all cellular components of the airway wall $(77,78)$. Postmortem studies reveal that airway wall thickening, due in part to subepithelial fibrosis, is present in asthmatic patients, and this observation seems to correlate with the severity of airway hyperresponsiveness and airflow obstruction $(79,80)$. Collagen deposition in the airway subepithelial space is essentially an inappropriate wound healing response due to chronic inflammation in the airway and is characterized by the activation of cells that form the structural and support elements of the airway, including epithelial cells, smooth muscle cells, fibroblasts, and endothelial cells $(81,82)$. One consequence of this fibrotic response is that it creates an ECM-rich environment that favors the survival and persistence of recruited immune cells around the asthmatic airway, thereby contributing to the chronicity and severity of the disease.

Triggers for allergic and asthmatic diseases are many and varied, but growing data suggests that exuberant fungal growth associated with dwellings, schools, and places of employment present a growing health hazard to individuals in all age groups (83). Pulmonary responses to Aspergillus fumigatus spores and conidia span a wide clinical spectrum, including rapid clearance of the conidia without lung injury, allergic lung disease with or without fungus colonization (84), and lung destruction due to invasive pulmonary aspergillosis (85). The coordinated activities of alveolar macrophages, infiltrating platelets, and infiltrating neutrophils effectively prevent the retention of $A$. fumigatus in the airways of immunocompetent individuals $(86,87)$, but allergic responses to $A$. fumigatus involve a number of immunologic abnormalities, including elevated levels of IgE, increased amounts of Th2 cytokines (88), eosinophil- and T cell-mediated inflammation, and marked airway remodeling $(89,90)$. Experimentally, the allergic responseto $A$. fumigatus, which is dominated by Th2 cytokines and chemokines, markedly compromises neutrophil and macrophage function, allowing intact conidia and degraded fungal components to persist for many weeks in M2 macrophages from allergic mice (91). Strategies directed toward the elimination of fungal material in the lungs of allergic mice through attenuation of the Th2 inflammatory response (92-96) or promotion of M2 macrophage apoptosis $(97,98)$ have proved very effective at reversing established experimental fungal asthma and the airway remodeling associated with this model. Therefore, the allergic responses to A. fumigatus conidia observed in atopic individuals and asthmatics seems to promote chronic airway remodeling through a skewed immune response that fails to adequately clear this pathogen from the pulmonary system.

Parasites and their hosts both exploit the tissue fibrotic process for survival. One of the major pathological features of most multicellular parasites is their ability to persist and establish a chronic infection. This leads to chronic inflammation, which in turn can lead to severe fibrotic modification of infected tissues and organs. Parasite and host seem to both contribute to the fibrotic process.
This response is advantageous to the parasite because it walls it off from the host's immune system, thereby allowing the parasite to persist and survive in a hostile environment. Unlike the other fibrotic conditions described above, this response is also advantageous to the host because it walls off the offending secretory products produced by the parasites, thereby preventing them from modulating and suppressing the host's immune response.

Although several parasites have documented effects on the fibrotic response in a number of organs (Table 1), Schistosoma mansoni provides a well studied example of a multicellular parasite that induces prominent fibrotic responses in the alimentary system during the natural course of infection and in the pulmonary system when eggs from this parasite are administered i.v. in experimental models (reviewed in ref. 99). The early response to infection with $S$. mansoni is associated with the activation of Th1 cells, and this causes the production of large amounts of IL-1 $\beta$, TNF- $\alpha$, IFN- $\gamma$, and NO (100). The cytokine response eventually switches to a Th2 cytokine profile as eggs from this parasite are deposited and becomes dominated by IL-4, IL-5, IL-10, and IL-13 (101). Several cytokine-based intervention studies have shown that the Th1 cytokines (102) dampen the fibrotic response, whereas the Th2 cytokines $(103,104)$ and chemokines (32) promote this response. During various phases of schistosomiasis, tissue-resident macrophages are a major source of leukocyte chemotactic factors (105), cytokines (106), growth factors (107), and free radicals (108). However, prolonged exposure of macrophages to Th2 cytokines and Th2-associated factors during S. mansoni-induced immunopathology (109) induces macrophages to become alternatively activated (that is, to become M2 macrophages) (29). This ultimately leads to their involvement in the tissue destructive fibrotic response (99). In the Th2 cytokine environment of a parasitic infection, L-arginine is predominately metabolized by ARG1, which is highly expressed by M2 macrophages (108). L-arginine metabolism by ARG1 generates urea and L-ornithine, which leads to the production of polyamines and proline, factors that exert prominent effects on cell growth; to collagen formation; and ultimately to tissue fibrosis $(110,111)$. More recently, M2 macrophages have been shown to be essential for host survival during the chronic inflammatory response to infection with $S$. mansoni, because in their complete absence the egg-induced inflammatory response is lethal (112). Therefore, M2 macrophages exert a prominent role in the profibrotic response during persistent infection with the parasite S. mansoni and possibly in similar fibrotic responses during infection with other parasites such as Cryptosporidium parvum $(113,114)$.

\section{Summary and clinical therapeutic considerations}

The magnitude and intensity of the immune response against an infective pathogen dictates the course and, therefore, the outcome of the infection. The virulence and survival of microorganisms in host tissues depend on their evasion of the immune system. The bidirectional interaction between the immune system and the pathogen is characterized by a dynamic balancing act between host mechanisms aimed at promoting tissue repair and pathogen strategies keenly tuned toward survival and persistence. The importance of this balance is highlighted by the Th2-type cytokine inflammatory response, which is aimed to repair tissue injury that results from viral and bacterial infection, but this cytokine environment can be exploited by and allow the persistence of these pathogens. Our major point is that this persistence might ultimately lead to debilitating 
tissue fibrosis. The fibrotic response might also be directly elicited by the response of activated fibroblasts to pathogen byproducts acting through PRRs and possibly other PAMP-detection mechanisms. Lytic and latent virus infection leads to major tissue injury and fibrosis both directly and possibly through the expression of viral gene products such as vGPCR in immune and structural cells within various organ sites, including the lung. The persistence of pathogens and/or their byproducts, such as those associated with fungal and multicellular parasite infections, also precipitates a fibrotic process. The exact mechanism(s) leading to fibrotic changes in the context of fungal and parasite infection is not presently clear, but both types of infection share the common feature of a demonstrable skewing of the cytokine response toward a Th2-type cytokine profile, thereby leading to the increased presence and activity of fibroblasts of various origins (that is, derived from resident cells, BM cells, or epithelial-mesenchymal transition) and M2 macrophages (Figure 1). Although the fibrotic response during nearly all challenges with a pathogen is often mediated by Th2 activation or activity, it is now clear that the complete absence of the Th2-mediated fibrotic response is not always desirable, in that its absence can increase the susceptibility of the host to the deleterious effects of parasitism.

The hallmark therapy for chronic inflammation is the group of immunosuppressive agents known as corticosteroids. Clearly, suppressing the immune response is unlikely to be a good approach to preventing the fibrotic tissue damage that occurs during chronic inflammation if, as seems increasingly probable, the stimulus for fibrosis is provided by the byproducts of these infectious agents or by cells such as M2 macrophages and activated fibroblasts, both of which are unaffected by this form of therapy. Alternatively, strategies directed toward eliminating these byproducts and/or their effects might prove highly beneficial in the context of fibrosis during chronic inflammation, as their elimination would presumably diminish the concomitant chronic inflammatory and fibrotic mechanisms. Therefore, increased understanding of the importance of the interplay between pathogens and their byproducts in the tissue healing process might provide novel treatment interventions in chronic fibrosing diseases in numerous organ systems.

\section{Acknowledgments}

The authors thank Steven L. Kunkel for helpful discussions and Robin G. Kunkel for her outstanding artistic contributions to this Review. We apologize for the inadvertent omission of any published work pertinent to the topic of this Review. Funding provided by NIH grants R01 HL69865, R01 HL073728, and P50 HL56402 (to C.M. Hogaboam) supported this work.

Address correspondence to: Cory M. Hogaboam, Room 4057 BSRB, 109 Zina Pitcher Place, Ann Arbor, Michigan 481092200, USA. Phone: (734) 936-7854; Fax: (734) 936-7996; E-mail: Hogaboam@umich.edu.
1. Wynn, T.A. 2004. Fibrotic disease and the $\mathrm{T}(\mathrm{H}) 1 /$ $\mathrm{T}(\mathrm{H}) 2$ paradigm. Nat. Rev. Immunol. 4:583-594.

2. Raghow, R. 1994. The role of extracellular matrix in post-inflammatory wound healing and fibrosis. FASEB J. 8:823-831.

3. Gharaee-Kermani, M., and Phan, S.H. 2001. Role of cytokines and cytokine therapy in wound healing and fibrotic diseases. Curr. Pharm. Des. 7:1083-1103.

4. Coelho, A.L., Hogaboam, C.M., and Kunkel, S.L. 2005. Chemokines provide the sustained inflammatory bridge between innate and acquired immunity. Cytokine Growth Factor Rev. 16:553-560.

5. Mutsaers, S.E., Bishop, J.E., McGrouther, G., and Laurent, G.J. 1997. Mechanisms of tissue repair: from wound healing to fibrosis. Int. J. Biochem. Cell Biol. 29:5-17.

6. Chambers, R.C., Laurent, G.J., and WestergrenThorsson, G. 1998. Cadmium inhibits proteoglycan and procollagen production by cultured human lung fibroblasts. Am. J. Respir. Cell Mol. Biol. 19:498-506.

7. Gharaee-Kermani, M., Nozaki, Y., Hatano, K., and Phan, S.H. 2001. Lung interleukin-4 gene expression in a murine model of bleomycin-induced pulmonary fibrosis. Cytokine. 15:138-147.

8. McCawley, L.J., and Martrisian, L.M. 2001. Matrix metalloproteinases: they're not just for matrix anymore! Curr. Opin. Cell Biol. 13:534-540.

9. Chodon, T., et al. 2000. Keloid-derived fibroblasts are refractory to Fas-mediated apoptosis and neutralization of autocrine transforming growth factor-beta 1 can abrogate this resistance. Am. J. Pathol. 157:1661-1669.

10. Jiang, D., et al. 2005. Regulation of lung injury and repair by Toll-like receptors and hyaluronan. Nat. Med. 11:1173-1179.

11. Dunn, M.A., et al. 1977. Liver collagen synthesis in murine schistosomiasis. J. Clin. Invest. 59:666-674.

12. Jakubzick, C., et al. 2003. Impact of interleukin-13 responsiveness on the synthetic and proliferative properties of Th1- and th2-type pulmonary granuloma fibroblasts. Am. J. Pathol. 162:1475-1486.

13. Sato, J., et al. 2003. The fibrinolytic system in dissemination and matrix protein deposition during a mycobacterium infection. Am. J. Pathol. 163:517-531.

14. Friedman, S.L. 2004. Mechanisms of disease: mechanisms of hepatic fibrosis and therapeutic implications. Nat. Clin. Pract. Gastroenterol. Hepatol. 1:98-105.

15. Takeda, K., Kaisho, T., and Akira, S. 2003. Toll-like receptors. Annu. Rev. Immunol. 21:335-376.

16. Buckley, C.D., et al. 2001. Fibroblasts regulate the switch from acute resolving to chronic persistent inflammation. Trends Immunol. 22:199-204.

17. Smith, R.S., Smith, T.J., Blieden, T.M., and Phipps, R.P. 1997. Fibroblasts as sentinel cells. Synthesis of chemokines and regulation of inflammation. Am. J. Pathol. 151:317-322.

18. Zhang, K., Rekhter, M.D., Gordon, D., and Phan, S.H. 1994. Myofibroblasts and their role in lung collagen gene expression during pulmonary fibrosis. A combined immunohistochemical and in situ hybridization study. Am. J. Pathol. 145:114-125.

19. Sempowski, G.D., Borrello, M.A., Blieden, T.M., Barth, R.K., and Phipps, R.P. 1995. Fibroblasts heterogeneity in the healing wound. Wound Repair Regen. 3:120-131.

20. Derdak, S., et al. 1992. Differential collagen and fibronectin production by Thy-1+ve and Thy-1-ve fibroblast subpopulations. Am. J. Physiol. 263:L283-L290.

21. Rinn, J.L., et al. 2006. Anatomic demarcation by positional variation in fibroblast gene expression programs. PLoS Genet. 2:e119.

22. Parsonage, G., et al. 2005. A stromal address code defined by fibroblasts. Trends Immunol. 26:150-156.

23. Chapman, H.A. 2004. Disorders of lung matrix remodeling. J. Clin. Invest. 113:148-157. doi:10.1172/JCI200420729.

24. Bucala, R., et al. 1994. Circulating fibrocytes define a new leukocyte subpopulation that mediates tissue repair. Mol. Med. 1:71-81.

25. Chesney, J., Bacher, M., Bender, A., and Bucala, R. 1997. The peripheral blood fibrocyte is a potent antigen-presenting cell capable of priming naive T cells in situ. Proc. Natl. Acad. Sci. U. S. A. 94:6307-6312.

26. Phillips, R.J., et al. 2004. Circulating fibrocytes traf- fic to the lungs in response to CXCL12 and mediate fibrosis. J. Clin. Invest. 114:438-446. doi:10.1172/ JCI200420997.

27. Moore, B.B., et al. 2005. CCR2-mediated recruitment of fibrocytes to the alveolar space after fibrotic injury. Am. J. Pathol. 166:675-684.

28. Quan, T.E., et al. 2004. Circulating fibrocytes: collagen-secreting cells of the peripheral blood. Int. J. Biochem. Cell Biol. 36:598-606.

29. Gordon, S. 2003. Alternative activation of macrophages. Nat. Rev. Immunol. 3:23-35.

30. Mantovani, A., et al. 2004. The chemokine system in diverse forms of macrophage activation and polarization. Trends Immunol. 25:677-686.

31. Raes, G., et al. 2002. Differential expression of FIZZ1 and $\mathrm{Ym} 1$ in alternatively versus classically activated macrophages. J. Leukoc. Biol. 71:597-602.

32. Jakubzick, C., et al. 2004. Role of CCR4 ligands, CCL17 and CCL22, during Schistosoma mansoni egginduced pulmonary granuloma formation in mice. Am. J. Pathol. 165:1211-1221.

33. Belperio, J.A., et al. 2004. The role of the Th2 CC chemokine ligand CCL17 in pulmonary fibrosis. J. Immunol. 173:4692-4698.

34. Creagh, E.M., and O'Neill, L.A. 2006. TLRs, NLRs and RLRs: a trinity of pathogen sensors that cooperate in innate immunity. Trends Immunol. 27:352-357.

35. Akira, S., Takeda, K., and Kaisho, T. 2001. Tolllike receptors: critical proteins linking innate and acquired immunity. Nat. Immunol. 2:675-680.

36. Pasare, C., and Medzhitov, R. 2005. Toll-like receptors: linking innate and adaptive immunity. $A d v$. Exp. Med. Biol. 560:11-18.

37. Martinon, F., and Tschopp, J. 2005. NLRs join TLRs as innate sensors of pathogens. Trends Immunol. 26:447-454.

38. Kato, H., et al. 2005. Cell type-specific involvement of RIG-I in antiviral response. Immunity. 23:19-28.

39. Akira, S., and Takeda, K. 2004. Toll-like receptor signalling. Nat. Rev. Immunol. 4:499-511.

40. Akira, S., Uematsu, S., and Takeuchi, O. 2006. Pathogen recognition and innate immunity. Cell. 124:783-801. 
41. Rifkin, I.R., et al. 2005. Toll-like receptors, endogenous ligands, and systemic autoimmune disease. Immunol. Rev. 204:27-42.

42. Binder, R.J., Vatner, R., and Srivastava, P. 2004. The heat-shock protein receptors: some answers and more questions. Tissue Antigens. 64:442-451.

43. Cosma, C.L., Sherman, D.R., and Ramakrishnan, L. 2003. The secret lives of the pathogenic mycobacteria. Annu. Rev. Microbiol. 57:641-676.

44. Mantovani, A., Allavena, P., and Sica, A. 2004. Tumour-associated macrophages as a prototypic type II polarised phagocyte population: role in tumour progression. Eur. J. Cancer. 40:1660-1667.

45. Katakura, T., et al. 2004. CCL17 and IL-10 as effectors that enable alternatively activated macrophages to inhibit the generation of classically activated macrophages. J. Immunol. 172:1407-1413.

46. Matzinger, P. 1994. Tolerance, danger, and the extended family. Annu. Rev. Immunol. 12:991-1045.

47. Droemann, D., et al. 2003. Toll-like receptor 2 is expressed by alveolar epithelial cells type II and macrophages in the human lung. Histochem. Cell Biol. 119:103-108.

48. Morris, G.E., et al. 2005. Agonists of Toll-like receptors 2 and 4 activate airway smooth muscle via mononuclear leukocytes. Am. J. Respir. Crit. Care Med. 171:814-822.

49. Greene, C.M., et al. 2005. TLR-induced inflammation in cystic fibrosis and non-cystic fibrosis airway epithelial cells. J. Immunol. 174:1638-1646.

50. Otte, J.M., Rosenberg, I.M., and Podolsky, D.K. 2003. Intestinal myofibroblasts in innate immune responses of the intestine. Gastroenterology. 124:1866-1878.

51. Keane, M.P., et al. 1997. The CXC chemokines, IL-8 and IP-10, regulate angiogenic activity in idiopathic pulmonary fibrosis. J. Immunol. 159:1437-1443.

52. Pierer, M., et al. 2004. Chemokine secretion of rheumatoid arthritis synovial fibroblasts stimulated by Toll-like receptor 2 ligands. J. Immunol. 172:1256-1265.

53. Shishido, T., et al. 2003. Toll-like receptor-2 modulates ventricular remodeling after myocardial infarction. Circulation. 108:2905-2910.

54. See, F., et al. 2005. Fibrosis as a therapeutic target post-myocardial infarction. Curr. Pharm. Des. 11:477-487.

55. Proost, P., et al. 2004. Synergistic induction of CXCL9 and CXCL11 by Toll-like receptor ligands and interferon-gamma in fibroblasts correlates with elevated levels of CXCR3 ligands in septic arthritis synovial fluids. J. Leukoc. Biol. 75:777-784.

56. Rudd, B.D., et al. 2005. Differential role for TLR3 in respiratory syncytial virus-induced chemokine expression. J. Virol. 79:3350-3357.

57. Anders, H.J., et al. 2004. Activation of toll-like receptor-9 induces progression of renal disease in MRL-Fas(lpr) mice. FASEB J. 18:534-536.

58. Fischer, S.F., et al. 2005. Toll-like receptor 9 signaling can sensitize fibroblasts for apoptosis. Immunol. Lett. 97:115-122.

59. Hasan, U.A., Trinchieri, G., and Vlach, J. 2005. Toll-like receptor signaling stimulates cell cycle entry and progression in fibroblasts. J. Biol. Chem. 280:20620-20627.

60. Lloyd, C.M., et al. 1997. RANTES and monocyte chemoattractant protein-1 (MCP-1) play an important role in the inflammatory phase of crescentic nephritis, but only MCP-1 is involved in crescent formation and interstitial fibrosis. J. Exp. Med. 185:1371-1380.

61. Kolattukudy, P.E., et al. 1998. Myocarditis induced by targeted expression of the MCP-1 gene in murine cardiac muscle. Am. J. Pathol. 152:101-111.

62. Antoniades, H.N., et al. 1992. Expression of monocyte chemoattractant protein $1 \mathrm{mRNA}$ in human idiopathic pulmonary fibrosis. Proc. Natl. Acad. Sci. U. S. A. 89:5371-5375.
63. Kleiner, D.E. 2005. The liver biopsy in chronic hepatitis C: a view from the other side of the microscope. Semin. Liver Dis. 25:52-64.

64. Martinez-Hernandez, A., and Amenta, P.S. 1995 The extracellular matrix in hepatic regeneration. FASEB J. 9:1401-1410.

65. Bedossa, P., et al. 1993. Transforming growth factor beta 1: in situ expression in the liver of patients with chronic hepatitis $\mathrm{C}$ treated with alpha interferon. Gut. 34(2 Suppl.):S146-S147.

66. Okuda, M., et al. 2002. Mitochondrial injury, oxidative stress, and antioxidant gene expression are induced by hepatitis $\mathrm{C}$ virus core protein. Gastroenterology. 122:366-375.

67. Marshall, A., et al. 2005. Relation between hepatocyte $\mathrm{G} 1$ arrest, impaired hepatic regeneration, and fibrosis in chronic hepatitis $\mathrm{C}$ virus infection. Gastroenterology. 128:33-42.

68. Kunkel, S.L., Lukacs, N.W., Strieter, R.M., and Chensue, S.W. 1996. Th1 and Th2 responses regulate experimental lung granuloma development. Sarcoidosis Vasc. Diffuse Lung Dis. 13:120-128.

69. Mora, A.L., et al. 2005. Lung infection with gamma herpesvirus induces progressive pulmonary fibrosis in Th2 biased mice. Am. J. Physiol. Lung Cell. Mol. Physiol. 289:L711-L721.

70. Tang, Y.W., et al. 2003. Herpesvirus DNA is consistently detected in lungs of patients with idiopathic pulmonary fibrosis. J. Clin. Microbiol. 41:2633-2640.

71. Prasse, A., et al. 2006. A vicious circle of alveolar macrophages and fibroblasts perpetuates pulmonary fibrosis via CCL18. Am.J. Respir. Crit. Care Med. 173:781-792.

72. Mora, A.L., et al. 2006. Activation of alveolar macrophages via the alternative pathway in herpesvirus-induced lung fibrosis. Am. J. Respir. Cell Mol. Biol. 35:466-473.

73. Ebrahimi, B., Dutia, B.M., Brownstein, D.G., and Nash, A.A. 2001. Murine gammaherpesvirus-68 infection causes multi-organ fibrosis and alters leukocyte trafficking in interferon-gamma receptor knockout mice. Am. J. Pathol. 158:2117-2125.

74. Lok, S.S., et al. 2002. Murine gammaherpes virus as a cofactor in the development of pulmonary fibrosis in bleomycin resistant mice. Eur. Respir. J. 20:1228-1232.

75. Holst, P.J., et al. 2001. Tumorigenesis induced by the HHV8-encoded chemokine receptor requires ligand modulation of high constitutive activity. J. Clin. Invest. 108:1789-1796. doi:10.1172/ JCI200113622.

76. Smit, M.J., et al. 2002. Kaposi's sarcoma-associated herpesvirus-encoded $\mathrm{G}$ protein-coupled receptor ORF74 constitutively activates p44/p42 MAPK and Akt via G(i) and phospholipase C-dependent signaling pathways. J. Virol. 76:1744-1752.

77. Fahy, J.V., Corry, D.B., and Boushey, H.A. 2000. Airway inflammation and remodeling in asthma. Curr. Opin. Pulm. Med. 6:15-20.

78. Elias, J.A., Zhu, Z., Chupp, G., and Homer, R.J. 1999. Airway remodeling in asthma. J. Clin. Invest. 104:1001-1006.

79. Niimi, A., et al. 2000. Airway wall thickness in asthma assessed by computed tomography. Relation to clinical indices. Am. J. Respir. Crit. Care Med. 162:1518-1523.

80. Djukanovic, R. 2000. Asthma: a disease of inflammation and repair. J. Allergy Clin. Immunol. 105:522-526

81. Bento, A.M., and Hershenson, M.B. 1998. Airway remodeling: potential contributions of subepithelial fibrosis and airway smooth muscle hypertrophy/hyperplasia to airway narrowing in asthma. Allergy Asthma Proc. 19:353-358.

82. Vignola, A.M., et al. 2000. Structural consequences of airway inflammation in asthma. J. Allergy Clin. Immunol. 105:514-517.

83. Kurup, V.P., Shen, H.D., and Banerjee, B. 2000. Respi- ratory fungal allergy. Microbes Infect. 2:1101-1110.

84. Schwartz, H.J., and Greenberger, P.A. 1991. The prevalence of allergic bronchopulmonary aspergillosis in patients with asthma, determined by serologic and radiologic criteria in patients at risk. J. Lab. Clin. Med. 117:138-142.

85. Elstad, M.R. 1991. Aspergillosis and lung defenses. Semin. Respir. Infect. 6:27-36.

86. Schaffner, A., Douglas, H., and Braude, A. 1982. Selective protection against conidia by mononuclear and against mycelia by polymorphonuclear phagocytes in resistance to Aspergillus: observations on these two lines of defense in vivo and in vitro with human and mouse phagocytes. J. Clin. Invest. 69:617-625.

87. Christin, L., et al. 1998. Human platelets damage Aspergillus fumigatus hyphae and may supplement killing by neutrophils. Infect. Immun. 66:1181-1189.

88. Skov, M., Poulsen, L.K., and Koch, C. 1999. Increased antigen-specific Th- 2 response in allergic bronchopulmonary aspergillosis (ABPA) in patients with cystic fibrosis. Pediatr. Pulmonol. 27:74-79.

89. Knutsen, A.P., et al. 1994. Asp f I CD4+ TH2-like Tcell lines in allergic bronchopulmonary aspergillosis. J. Allergy Clin. Immunol. 94:215-221.

90. Greenberger, P.A., and Patterson, R. 1987. Allergic bronchopulmonary aspergillosis. Model of bronchopulmonary disease with defined serologic, radiologic, pathologic and clinical findings from asthma to fatal destructive lung disease. Chest. 91 (6 Suppl.):165S-171S.

91. Hogaboam, C.M., et al. 2000. Chronic airway hyperreactivity, goblet cell hyperplasia, and peribronchial fibrosis during allergic airway disease induced by Aspergillus fumigatus. Am. J. Pathol. 156:723-732.

92. Blease, K., et al. 2001. Therapeutic effect of IL-13 immunoneutralization during chronic experimental fungal asthma. J. Immunol. 166:5219-5224.

93. Schuh, J.M., et al. 2002. Airway hyperresponsiveness, but not airway remodeling, is attenuated during chronic pulmonary allergic responses to Aspergillus in CCR4-/- mice. FASEB J. 16:1313-1315.

94. Blease, K., et al. 2000. Airway remodeling is absent in CCR1-/- mice during chronic fungal allergic airway disease. J. Immunol. 165:1564-1572.

95. Carpenter, K.J., et al. 2005. Therapeutic targeting of CCR1 attenuates established chronic fungal asthma in mice. Br.J. Pharmacol. 145:1160-1172.

96. Hogaboam, C.M., et al. 2005. The therapeutic potential in targeting CCR5 and CXCR4 receptors in infectious and allergic pulmonary disease. Pharmacol. Ther. 107:314-328.

97. Blease, K., et al. 2001. IL-13 fusion cytotoxin ameliorates chronic fungal-induced allergic airway disease in mice. J. Immunol. 167:6583-6592.

98. Schuh, J.M., et al. 2003. Intrapulmonary targeting of RANTES/CCL5-responsive cells prevents chronic fungal asthma. Eur. J. Immunol. 33:3080-3090.

99. Wynn, T.A., Thompson, R.W., Cheever, A.W., and Mentink-Kane, M.M. 2004. Immunopathogenesis of schistosomiasis. Immunol. Rev. 201:156-167.

100.Chensue, S.W., et al. 1993. Evolving T cell responses in murine schistosomiasis. Th2 cells mediate secondary granulomatous hypersensitivity and are regulated by CD8+ T cells in vivo. J. Immunol. 151:1391-1400

101.Grzych,J.M., et al. 1991. Egg deposition is the major stimulus for the production of Th2 cytokines in murine schistosomiasis mansoni. J. Immunol. 146:1322-1327.

102. Wynn, T.A., et al. 1995. An IL-12-based vaccination method for preventing fibrosis induced by schistosome infection. Nature. 376:594-596.

103. Cheever, A.W., et al. 1994. Anti-IL-4 treatment of Schistosoma mansoni-infected mice inhibits development of $\mathrm{T}$ cells and non-B, non- $\mathrm{T}$ cells expressing Th2 cytokines while decreasing egg-induced 
hepatic fibrosis. J. Immunol. 153:753-759.

104. Chiaramonte, M.G., Donaldson, D.D., Cheever A.W., and Wynn, T.A. 1999. An IL-13 inhibitor blocks the development of hepatic fibrosis during a T-helper type 2-dominated inflammatory response. J. Clin. Invest. 104:777-785.

105.Lukacs, N.W., et al. 1995. Macrophage inflammatory protein-1 alpha influences eosinophil recruitment in antigen-specific airway inflammation. Eur. J. Immunol. 25:245-251.

106.Chensue, S.W., et al. 1995. In vivo regulation of macrophage IL-12 production during type 1 and type 2 cytokine-mediated granuloma formation. J. Immunol. 155:3546-3551.

107.Lukacs, N.W., et al. 1996. Stem cell factor (c-kit ligand) influences eosinophil recruitment and histamine levels in allergic airway inflammation. J. Immunol. 156:3945-3951.

108. Hesse, M., et al. 2001. Differential regulation of nitric oxide synthase- 2 and arginase- 1 by type 1 / type 2 cytokines in vivo: granulomatous pathology is shaped by the pattern of L-arginine metabolism. J. Immunol. 167:6533-6544.

109.Pearce, E.J., and MacDonald, A.S. 2002. The immunobiology of schistosomiasis. Nat. Rev. Immunol. 2:499-511.

110.Munder, M., Eichmann, K., and Modolell, M. 1998. Alternative metabolic states in murine macrophages reflected by the nitric oxide syn- thase/arginase balance: competitive regulation by CD4+ T cells correlates with Th1/Th2 phenotype. J. Immunol. 160:5347-5354

111. Goerdt, S., et al. 1999. Alternative versus classical activation of macrophages. Pathobiology. 67:222-226.

112.Herbert, D.R., et al. 2004. Alternative macrophage activation is essential for survival during schistosomiasis and down modulates $\mathrm{T}$ helper 1 responses and immunopathology. Immunity. 20:623-635.

113.Noel, W., et al. 2004. Alternatively activated macrophages during parasite infections. Trends Parasitol. 20:126-133.

114.Waters, W.R., et al. 1999. Cryptosporidium parvum-induced inflammatory bowel disease in TCR-beta- $x$ TCR-delta-deficient mice. J. Parasitol. 85:1100-1105.

115.Jeevan, A., et al. 1995. Ultraviolet radiation reduces phagocytosis and intracellular killing of mycobacteria and inhibits nitric oxide production by macrophages in mice. J. Leukoc. Biol. 57:883-890.

116.Schaaf, B., et al. 2000. Neutrophil inflammation and activation in bronchiectasis: comparison with pneumonia and idiopathic pulmonary fibrosis Respiration. 67:52-59.

117. Thung, S.N. 2006. Histologic findings in recurrent HBV. Liver Transpl. 12(11 Suppl. 2):S50-S53.

118.Glanville, A.R., et al. 2005. Intravenous ribavirin is a safe and cost-effective treatment for respiratory syncytial virus infection after lung transplantation.
J. Heart Lung Transplant. 24:2114-2119.

119.Lok, S.S., et al. 2001. Epstein-Barr virus and wild p53 in idiopathic pulmonary fibrosis. Respir. Med. 95:787-791.

120.Wynn, T.A., et al. 1995. IL-12 enhances vaccineinduced immunity to Schistosoma mansoni in mice and decreases T helper 2-cytokine expression, $\mathrm{IgE}$ production, and tissue eosinophilia. J. Immunol. 154:4701-4709.

121.Jakubzick, C., et al. 2002. Interleukin-13 fusion cytotoxin arrests Schistosoma mansoni egginduced pulmonary granuloma formation in mice. Am. J. Pathol. 161:1283-1297.

122.Fallon, P.G., Richardson, E.J., McKenzie, G.J., and McKenzie, A.N. 2000. Schistosome infection of transgenic mice defines distinct and contrasting pathogenic roles for IL-4 and IL-13: IL-13 is a profibrotic agent. J. Immunol. 164:2585-2591.

123. Benfield, T.L., et al. 1997. Alveolar damage in AIDSrelated Pneumocystis carinii pneumonia. Chest. 111:1193-1199.

124.Pinson, P., and Van der Straeten, M. 1991. Fibrotic stage of allergic bronchopulmonary candidiasis. Chest. 100:565-567.

125.Arora, S., et al. 2005. Role of IFN-gamma in regulating $\mathrm{T} 2$ immunity and the development of alternatively activated macrophages during allergic bronchopulmonary mycosis. J. Immunol. 174:6346-6356. 